\title{
A novel architecture of fibre-optic interferometric hydrophone
}

\author{
Xiaodi Huang, Desheng Chen* \\ Institute of Acoustics, Chinese Academy of Sciences, Beijing, China
}

\begin{abstract}
The fibre-optic interferometric hydrophone has been widely used in ocean acoustic applications. There are many different hydrophone systems in use. They can generally be classified as hull mounted, towed, or fixed (bottom mounted and vertical) array systems. Different optical architectures have evolved for each of the areas, which make a good case study on what aspects of a particular application influence the optical architecture. A novel architecture of fibre-optic hydrophone based on PMDI is theoretically and experimentally discussed in this paper. A novel optical configuration is proposed, and the modulation and demodulation system is built. A series of experiments are designed to analyse the characteristics of this system. The results of the experiments show that this type of fibre-optic interferometric hydrophone array has many advantages such as low noise, a large dynamic range.
\end{abstract}

\section{Introduction}

Time Division Multiplexing (TDM) has become the preferred multiplexing approach in fibre-optic interferometric hydrophone arrays. The major motivation is the high levels of multiplexing gain that can be achieved with TDM. There are a variety of approaches to TDM, and phase generated carrier (PGC) modulation scheme is one of the most popular modulation approaches. PGC modulation is usually realized by a laser which is directly modulated with a carrier [1,2]. However, the cost of tuneable laser source is high, and the bandwidth limitations and added noise of frequencymodulated laser source ultimately limit the multiplexing gains that can be achieved.

In this paper, a novel scheme of fibre-optic interferometric hydrophone array is proposed. A pathmatched differential interferometer (PMDI) is located on the receiver side, with a thin shell PZT ceramic cylinder as a phase modulator. Based on this configuration, a system of fibre-optic interferometric hydrophone array is built.

\section{The scheme of the array based on PMDI}

\subsection{PMDI with a PZT phase modulator}

As shown in Figure 1, a path-matched differential interferometer (PMDI) is located in the receiver side, the length difference of two arms of the PMDI is equal to that of the sensor head [3]. When the light returns to the PMDI, it is split to four light pulses. The optical path difference of two light pulses is zero, and thus these two light beams interfere. The output of the PMDI carries the signal from the sensor head.

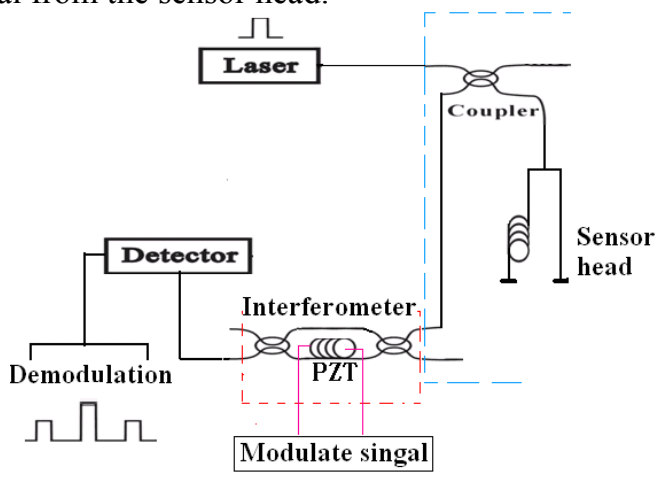

Fig. 1. PGC modulation based on PZT and PMDI.

The outputs of the PMDI can be expressed as

$$
\begin{gathered}
I_{0}=I_{1}+I_{2}+2 \sqrt{I_{1} I_{2}} \cos \left(\varphi_{\mathrm{s}}+\varphi_{\mathrm{n}}+\varphi_{0}\right)=A+B \cos \varphi \\
A=I_{1}+I_{2}, \quad B=2 \sqrt{I_{1} I_{2}}, \quad \varphi=\varphi_{\mathrm{s}}+\varphi_{\mathrm{n}}+\varphi_{0}
\end{gathered}
$$

where, $I_{1}$ and $I_{2}$ are respectively the laser intensity of the two arms of the fiber sensor, $\varphi_{s}$ is directly proportional to the strain in the fibre induced by the acoustic signal, $\varphi_{n}$ is the phase noise, and $\varphi_{0}$ is the phase of the initial difference of the two arms.

PGC modulation introduces a large amplitude phase shift at a frequency outside of the signal band so as to detect small signal phase shifts and eliminate signal fading caused by environmental drifts.

Based on the architecture mentioned above, for a sinusoidal phase modulated signal with angular

\footnotetext{
Corresponding author: cds@mail.ioa.ac.cn
} 
frequency $\omega$ and amplitude $C$ imposed on the fiber interferometer, the output signal can be expressed as:

$$
I_{0}=A+B \cos (C \cos \omega t+\varphi)
$$

The traditional PGC modulation approach is to rapidly change the frequency of the laser. For a $1550 \mathrm{~nm}$ semiconductor laser, this change is realized by changing the driving current, which causes a large fluctuation of the laser intensity and induced noise.

In this paper,the PGC modulation can be realized by fiber twinned on a PZT phase modulator in one arm. When one arm of the fiber sensor is modulated by a sinusoidal signal with frequency $\omega$, the optical path difference of the two arms can be expressed as:

$$
l=l_{0}+\Delta l \cos \omega t
$$

where $l_{0}$ is the initial optical path difference, and $\Delta l$ is the shift amplitude of optical path difference [4].

If we denote $C=2 \pi n v \Delta l / c$, the output signal can be expressed as:

$$
I_{0}=A+B \cos (C \cos \omega t+\varphi)
$$

and thus the PGC modulation is realized.

\subsection{Design of the array of based on PMDI}

As shown in Fig.1, a fibre-optic interferometric hydrophone is built. More sensor head can be linked to build a hydrophone array. Fig.2 shows a 8 -element array based on PMDI mentioned above.

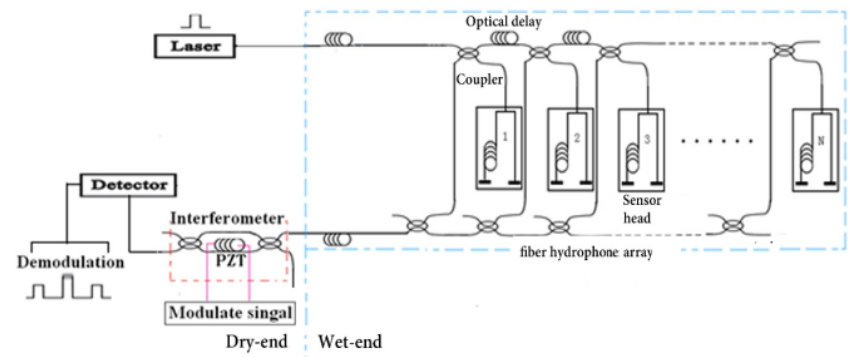

Fig. 2. 8-element array based on PMDI.

Furthermore, a PZT modulator is added to one arm of the compensatory interferometer, so the PGC external modulation can be realized. In the array the interrogation optical pulse is distributed by a coupler tree, which splits the optical power among all channels. Between each channel there is an optical delay, which is simply a section of optical fibre of the appropriate length. On the recombination side, another coupler tree is used to recombine and transport all outputs to the PMDI by a single fibre. Light beams from the same sensor head interfere through the PMDI.

This scheme has many advantages. The modulatable laser is not needful. Compared with the laser directly modulated with a carrier signal, the noise induced by the PZT phase modulator can be easily reduced. Moreover, there are fewer limitations on the bandwidth or dynamic range of the system.

\section{Experiments}

In our experiment, the first step is to study the characteristics of the system shown in Fig.3.

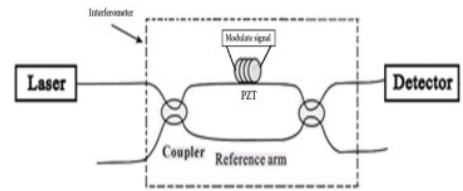

Fig. 3. PZT modulator in the interferometer.

A section of $30 \mathrm{~m}$ fiber is twinned on the PZT modulator. We modulate the PZT modulator with a sinusoidal signal $(12.5 \mathrm{kHz})$. As shown in Fig.4, the phase noise of the PZT modulator in interferometer is about $-100 \mathrm{~dB}$ at $1 \mathrm{kHz}$.

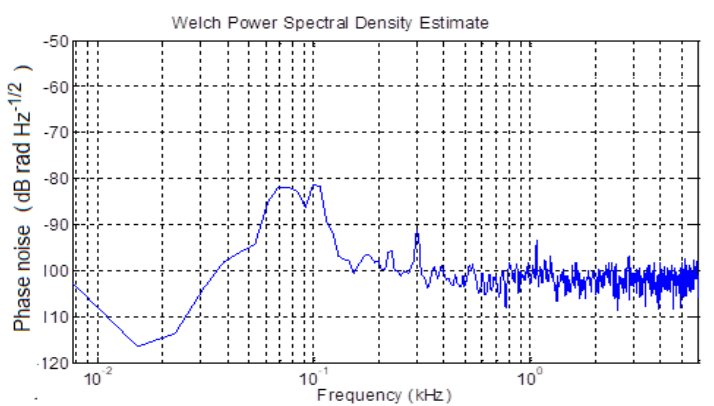

Fig. 4. Phase noise of the PZT modulator in the interferometer.
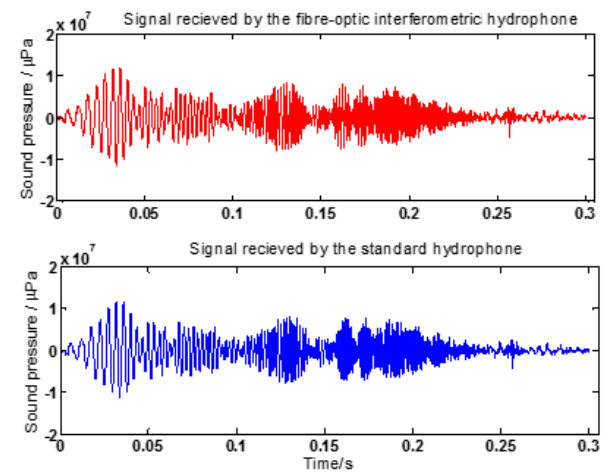

Fig. 5. Signal recieved by the fibre-optic interferometric hydrophone and the standard hydrophone.

We put the array shown in Fig.2 underwater and an underwater speaker emits a signal. Fig.5 shows the demodulated signal received by the fibre-optic hydrophone and the signal received by the standard hydrophone whose sensitivity is $-177 \mathrm{~dB}(0 \mathrm{~dB}$ ref. $1 \mathrm{~V} / \mu \mathrm{Pa})$. The correlation coefficient is 0.997 , and the pressure sensitivity of the fibre-optic hydrophone is $-130.7 \mathrm{~dB}(0 \mathrm{~dB}$ ref. $1 \mathrm{rad} / \mu \mathrm{Pa})$. The dynamic range of this system is roughly $120 \mathrm{~dB}$ at 1 $\mathrm{kHz}$.

\section{Conclusions}

A novel architecture of fibre-optic interferometric hydrophone is theoretically and experimentally discussed in this paper. It can be more widely used because of its good characteristics. Based on this architecture, we can develop different fibre-optic 
hydrophone arrays used in hull mounted, towed, or fixed array systems.

The work was supported by the Foundation of Chinese Academy of Sciences (No.6141A01051601) and IACAS Young Elite Researcher Project (No.QNYC201742). The authors wish to thank all the researchers participating in the experiment.

\section{References}

1. Dandridge A, Tveten A B, Kersey A D, Journal of Lightwave Technology. 5(7), 947- 952 (1987)

2. C. K. Kirkendall , A. Dandridge. J. Phys. D: Appl. Phys. 37, 197-216, (2004)

3. Lim T. K., Lin Y., Yip Y. M., Optics Communications. 159(4-6), 301-308 (1999)

4. W. W. Lin, S. T. Shih, M. H. Chen, S. C. Huang. Proceeding of SPIE - The International Society for Optical Engineering. 1814, 130-141 (1992) 